\title{
Hipermovilidad articular y Síndrome de Ehlers-Danlos: consideraciones desde el cuidado en enfermería
}

\author{
Francisca González-Adonis' ${ }^{1}$ Josephine Bratz² ${ }^{2}$ Marcelo Sandoval-Ramírez³, Camilo Guerrero-Nancuante ${ }^{4}$
}

\section{RESUMEN}

Los desórdenes del espectro hipermóvil y síndrome de Ehlers-Danlos (HSD y EDS por sus siglas en inglés) son enfermedades crónicas, consideradas extrañas y que afectan la calidad de vida de estos pacientes.

Según estudios internacionales, su prevalencia mundial se encuentra entre el 2 al 57 \% de la población, presentando, según la etapa de vida, manifestaciones clínicas variadas. Además, pueden ocurrir exacerbaciones de otras condiciones asociadas, por lo que se torna difícil diagnosticar y se generan múltiples consultas en los distintos niveles de atención.

En consecuencia, este escrito reflexiona respecto a la importancia del rol profesional de enfermería en la gestión del cuidado de niños y adultos que viven con este problema de salud, mediante una revisión de publicaciones actualizadas en torno a HSD y EDS.

Se enfatiza sobre cómo desde el cuidado de enfermería se puede acompañar a las personas con esta condición. Se concluye que es primordial tomar conciencia de la existencia de esta enfermedad de forma interdisciplinaria, para aportar en la pesquisa, el manejo y cuidado de las personas afectadas.

\section{PALABRAS CLAVE}

Atención de Enfermería; Inestabilidad de la Articulación; Síndrome de Ehlers-DanIos

\footnotetext{
1 Enfermera, estudiante de Magíster en Enfermería. Académica de la Escuela de Enfermería de la Universidad de Valparaíso-Chile.

2 Enfermera-Matrona. Magíster en Bioética, Fundación Vidya, Chile.

3 Fonoaudiólogo. Magíster en Bioética. Académico de la Escuela de Ciencias de la Salud, Universidad Viña del Mar-Chile.

4 Enfermero, estudiante de Magíster en Salud Pública. Académico de la Escuela de Enfermería, Universidad de Valparaíso-Chile.
}

Correspondencia: Francisca Jesús González-Adonis; francisca.gonzalez@uv.cl

Recibido: septiembre 26 de 2018

Aceptado: noviembre 26 de 2018

Cómo citar: González-Adonis F, Bratz J Sandoval-Ramírez M, Guerrero-Nancuante C. Hipermovilidad articular y síndrome de Ehlers-Danlos: consideraciones desde el cuidado en enfermería. latreia. 2019 0ct-Dic;32(4):346-353. D0I 10.17533/udea.iatreia.22. 


\section{SUMMARY}

Joint Hypermobility and Ehlers-Danlos Syndrome: Considerations from Nursing Care

Hypermobile spectrum disorders and Ehlers-Danlos syndrome (HSD and EDS) are chronic diseases, considered unusual and affecting the quality of life of these patients.

According to international studies, its prevalence worldwide is between 2 to $57 \%$ of the population and presenting, depending on the stage of life, varied clinical manifestations. Also, exacerbations of other associated conditions may occur, which makes it difficult to diagnose, generating several consults at the different levels of care.

Consequently, this paper reflects on the importance of the nurse professional role in managing the care of children and adults living with this health problem, through a review of updated publications on HSD and EDS.

Emphasis is placed on how nursing care can accompany people with this condition. It is concluded that it is essential to be aware of the existence of this pathology in an interdisciplinary way to contribute in the research, the management and care of the affected people.

\section{KEY WORDS}

Ehlers-Danlos Syndrome; Joint Instability; Nursing Care

\section{INTRODUCCIÓN}

Los HSD y EDS pertenecen a los desórdenes hereditarios de colágeno cuya prevalencia real no ha sido del todo establecida en la población chilena. Desde el punto de vista clínico y de calidad de vida, constituyen un desafío creciente para todos los profesionales que se desempeñan en las distintas áreas asistenciales de la salud. El polimorfismo de estas condiciones y las complicaciones asociadas, demandan del equipo de salud un exigente ejercicio interdisciplinario de pesquisa, diagnóstico, promoción y manejo. En general los diagnósticos de HSD y EDS son complejos de establecer $y$, a veces, las personas afectadas deambulan durante años y hasta décadas de médico en médico para conseguir el suyo ${ }^{(1)}$. Por el hecho de que los HSD/EDS son considerados patologías raras y que, en general, no se encuentran estudios que reflejen su real alcance, los expertos consideran que estas condiciones en su conjunto han sido por largo tiempo desatendidas $y$ tratadas de forma negligente ${ }^{(2)}$.

Lo necesario sería, en primer lugar, tomar nota de su existencia y en segundo lugar, entender que pueden significar para el individuo afectado una vida entera llena de sintomatología y complicaciones, llegando a comprometer sus actividades diarias, laborales, sociales y hasta amenazar su vida. Estas condiciones conllevan a la posibilidad de discapacidad, al afectar todos los ámbitos vitales.

EI propósito del presente texto es entregar una mirada general de los aspectos para tener en cuenta en el cuidado de enfermería de las personas con hipermovilidad y síndrome de Ehlers-Danlos, a partir de la revisión de la literatura. Además de motivar a la profundización sobre este problema emergente de salud, especialmente dentro del sistema público de salud chileno, el cual atiende al 80 \% de la población, sin descuidar la importancia de la coordinación en el sector privado.

\section{HIPERMOVILIDAD Y SÍNDROME DE EHLERS- DANLOS}

Hipermovilidad, o hiperlaxitud articular (HA), se define como la capacidad de una articulación de moverse en forma pasiva o activa más allá de los límites normales de sus ejes fisiológicos ${ }^{(3)}$. La hipermovilidad de un individuo puede constituir un diagnóstico aislado o formar parte de un conjunto de aspectos clínicos que sugieren la presencia de un síndrome, tal como ocurre en el caso de los EDS y en los recientemente definidos HSD ${ }^{(3)}$.

Cuando la hipermovilidad se observa en múltiples articulaciones se define como hipermovilidad articular generalizada (HAG), que está influenciada por la edad, la etnia y el sexo y podría ser asintomática. 
Sin embargo, se encuentra una creciente evidencia que indica la existencia de una serie de alteraciones músculo-esqueléticas y otras complicaciones sistémicas asociadas que pueden comprometer, de manera importante, la salud y la calidad de vida de las personas afectadas ${ }^{(2)}$.

Estudios señalan que en diferentes poblaciones en el mundo se han descrito prevalencias de HAG de entre el 2 y $57 \%{ }^{(4)}$. Este amplio rango se explica, probablemente, por el uso de diferentes métodos de evaluación clínica y las diversas clasificaciones e interpretaciones que de estos resulten; además, se ha advertido que puede existir un subdiagnóstico de personas hiperlaxas, cuya condición no llega a ser registrada en los sistemas de salud ${ }^{(4)}$.

La Sociedad Internacional de Síndrome de EhlersDanlos determinó en el año 2017 la utilización correcta del Beighton score como el procedimiento más adecuado para establecer el diagnóstico de HAG y, además, presentó una actualizada clasificación de HSD/EDS, describiendo 13 tipos de EDS y sus respectivos criterios clínicos ${ }^{(5)}$. El tipo de mayor prevalencia es el Hiperlaxo (hEDS), cuyo diagnóstico no dispone de un examen genético, por ende debe basarse en los criterios sugeridos ${ }^{(5)}$.

Dado que las alteraciones de salud que puede llegar a experimentar un individuo debido a su condición genética de HSD/hEDS son variables, probablemente progresivas y multisistémicas; en el momento de cuidar a personas con hiperlaxitud, el profesional enfermero debe comprender la complejidad de la condición y ser capaz de atender la gran variabilidad que puede presentarse entre los individuos afectados, considerando además los diversos factores sociales, culturales, políticos, económicos, entre otros, que la impactan de forma importante.

\section{CUIDADO DE ENFERMERÍA PARA LAS PERSONAS CON HIPERMOVILIDAD Y SÍNDROME DE EHLERS- DANLOS}

En el cuidado de enfermería, las personas que padecen HSD/hEDS deben ser miradas de manera integrada como portadoras de condición crónica que afecta prácticamente a todas las necesidades y esferas de vida del individuo. Se deben reconocer los detalles sintomatológicos en un cuadro integrado, no parcelado, como muchas veces les sucede a las personas atendidas.

La perspectiva fragmentada de la condición (por ejemplo según áreas de especialidad médica: reumatología, traumatología, neurología u otras) en muchas ocasiones demora el diagnóstico, lo que retrasa la posibilidad de un acompañamiento profesional en los procesos que vive el afectado y además, contribuye considerablemente al estrés del individuo.

Muchos pacientes al final son calificados como psiquiátricos, hipocondriacos o simplemente tratados erróneamente y nunca comprenden la propia enfermedad en su totalidad ${ }^{(2)}$. Cada afectado requiere de un alineamiento de cuidado individualizado e interdisciplinario, con atención a los detalles individuales en el plano físico, sicológico, social y espiritual. Asimismo, el cuidado necesita considerar el efecto de la enfermedad en el núcleo familiar, del cual, como en la mayoría de los casos de enfermedades crónicas, emerge la figura de un cuidador.

Lo primordial es reconocer la existencia de la problemática en las diferentes etapas de la vida y según las áreas de cuidados específicos. Para comprender la problemática y establecer lineamientos del cuidado, parece útil considerar la propuesta de progresión de hEDS establecida por Castori et al., la cual pretende ayudar a la elaboración de estrategias de prevención e intervención interdisciplinaria ${ }^{\left({ }^{6}\right)}$. Estos autores proponen tres fases progresivas, distribuidas en tres décadas de vida que, mostrando una evolución de signos y síntomas, ayudan a visualizar las complicaciones de estas condiciones en las diferentes etapas de la vida y a reconocer los momentos clave de intervención de enfermería mediante una valoración acuciosa ${ }^{(6)}$.

La primera fase ocurre entre la infancia y adolescencia, pudiendo aparecer los siguientes siognos y síntomas: estrías precoces, luxaciones/subluxaciones, los así llamados "dolores de crecimiento", dolores ocasionales de espalda y articulaciones, calambres, mialoias pos-ejercicio, hipotonía suave, retraso en el desarrollo psicomotor (DSM), problemas de coordinación sensoriomotora, cefaleas ocasionales o recurrentes, fácil fatigabilidad, estreñimiento o diarrea, hiperactividad bronquial, hipersensibilidad alimentaria y alteraciones en la función vesical. En esta fase, la intervención 
debe focalizarse en la promoción de estilos de vida saludables y ejercicios individualizados ${ }^{(6)}$.

Las manifestaciones durante la segunda fase, que comprende la segunda y tercera década de la vida, serían: artralgias, dolores de espalda, tenosinovitis, osteoartritis/espondilosis, osteopenia, mialgias, hiperalgesia muscular, caídas frecuentes, disfagia, disfonía, parestesias, cefaleas, síntomas de patología cervical, deficiente calidad del sueño, disnea posejercicio, irregularidades menstruales, dismenorrea, dispareunia/vulvodinia $\searrow$ desórdenes gastrointestinales. En consecuencia, se recomienda la promoción de estilos de vida saludables, ejercicio, fisioterapia y psicoterapia ${ }^{(6)}$. Ya en la tercera fase, es decir entre la tercera y cuarta década de la vida, aparecen de forma crónica las manifestaciones que incapacitan: artralgias, dolores de espalda, degeneraciones de los tendones y ligamentos, rigidez generalizada, osteoporosis, mialgias, fibromialgia, fatiga muscular generalizada, alodinia, reacciones anormales a estímulos sensoriales, cefalea, síntomas de compromiso cervical, malestar pos-ejercicio, fatiga matutina incapacitante, prolapso pélvico, incontinencia, prolapsos viscerales múltiples, insuficiencia pulmonar crónica y cistitis intersticial.

En esta última fase, tal como se mencionó anteriormente, las recomendaciones son la promoción de estilos de vida saludables, el ejercicio, la fisioterapia, la psicoterapia y los enfoques de medicina complementaria ${ }^{(6)}$.

Desde el punto de vista de la enfermería, y a la par con la progresión sintomática, se visualiza un aumento de necesidades de cuidado, las cuales deben coordinarse en cuanto a pesquisa, diagnóstico, promoción, manejo y mantención del estado, considerando a cada afectado según su individualidad y de acuerdo con la etapa de la vida.

Dada la gran variabilidad clínica en los individuos afectados, puede presentarse discapacidad en la primera o segunda etapa. Por lo anterior, se hace imperativo una pesquisa, diagnóstico e intervención oportunos, acogiendo las sugerencias de algunos expertos que señalan que la discapacidad grave pudo haber sido evitada en muchos casos ${ }^{(2)}$. Por tanto, en busca de un diagnóstico temprano, el profesional enfermero debe considerar en la valoración de enfermería las manifestaciones descritas anteriormente, comparándolas según la etapa de vida de la persona.

No obstante, en muchas ocasiones la pesquisa se presenta justamente de manera tardía y el cuidado debe enfocarse en aportar al retraso de la progresión y a paliar las manifestaciones. A causa del polimorfismo de los HSD y hEDS, su pesquisa podría ser requerida en todos los niveles asistenciales del sistema de salud. En eI nivel primario la problemática puede aparecer en los controles de salud infantil, de enfermedades crónicas y consultas de morbilidad; asimismo, en atenciones odontológicas, de maternidad y, quizás, especialmente en las de salud mental, ya que muchos afectados están en situación de policonsultantes, acudiendo de profesional en profesional hasta que alguien declare que la sintomatología es causada por depresión ${ }^{(2)}$, y que «en el fondo, el problema es psiquiátrico". En el nivel secundario, la posibilidad de pesquisar personas hiperlaxas está presente prácticamente en todas las especialidades, además de ser el nivel donde se realizan exámenes específicos y se debiese establecer el diagnóstico.

Los profesionales médicos que trabaja con personas con HSD/hEDS son principalmente reumatólogos, fisiatras, psiquiatras, neurólogos y traumatólogos, entre otros. Finalmente, a nivel terciario, se deberán enfrentar las complicaciones ocasionadas por las diversas consecuencias que tienen las alteraciones del tejido conectivo en el organismo, incluso las que por su gravedad requieran cuidados intensivos.

La pesquisa mediante anamnesis individual y familiar, como también mediante el examen físico exhaustivo y el screening de hipermovilidad, son esenciales. Una vez levantada la sospecha diagnóstica, será rol de enfermería velar por una continuidad del cuidado de las personas afectadas mediante la red asistencial, lo que involucra, en primer lugar, gestionar los recursos que permitan el diagnóstico y un manejo integrado, asimismo, aportar a la prevención de las complicaciones y la promoción de estilos de vida acordes con la conđición.

Dadas las características transgeneracionales de los HSD y hEDS, durante el cuidado individual debe considerarse que, al ser hereditarios, afectan a varias generaciones dentro de una misma familia, comprometiendo a varios integrantes. 
Lo anterior puede llevar a una aceptación de los síntomas, ya que "a todos les ha pasado lo mismo" $y$, por ende, muchas veces los síntomas no son vistos como problemáticos $y$ no motivan a consultar por la salud. Aquí se mezclan, adicionalmente, variables socioculturales $y$ de género respecto a las vivencias de dolor y resistencia física, pudiendo darse francas negaciones de las experiencias personales de los integrantes afectados.

Podría darse una incomprensión y hasta invisibilización de la experiencia intrafamiliar. Asimismo, pueden encontrarse problemas familiares graves, tanto relacionales como económicos, dada la existencia de varios integrantes enfermos o discapacitados a la vez, viéndose afectadas las expectativas del proyecto común de la vida familiar. Por lo expuesto anteriormente, los pilares del cuidado en todas las etapas de la vida, tanto individual como familiar, deben basarse en la comprensión y compasión por la experiencia personal del afectado $y$ no solo en la observabilidad de las manifestaciones. También es necesario el acompañamiento en los procesos de adaptación a la enfermedad crónica, como en la promoción del autocuidado y del empoderamiento ${ }^{(6)}$.

para cada fase progresiva, el cuidado de enfermería debe considerar características particulares de cada etapa de la vida, las que se ven interferidas por la condición de HSD/hEDS ${ }^{(5)}$. En este sentido, el profesional de enfermería que participa del cuidado de familias con niños percibirá a la infancia como un periodo de exploración, movimiento y juego activo, no esperando encontrarse con un niño que presente lesiones frecuentes, torpeza, caídas, hasta desmayos o que experimente dolor, y más aún, dolor crónico; pudiendo llegar a pasarse por alto una serie de manifestaciones clínicas relevantes al no diferenciarlas de las situaciones esperadas en la niñez.

Al considerar las manifestaciones músculo-esqueléticas que pueden darse durante esta etapa, la evaluación del desarrollo psicomotor (DSM) en un lactante en el control de salud infantil debe focalizarse en la pesquisa de hipotonía sin causa neurológica, dificultades en el control cefálico, aumento del esfuerzo para levantar brazos y dar patadas, estancamiento en vuelco, retraso en el desarrollo de las actividades finas, en el comienzo del balbuceo, arrastre en vez de gateo o retraso en la marcha, entre otros.
Estas manifestaciones pueden determinar un retraso en el DSM, ante el cual, habitualmente, se educaría respecto a la estimulación interdisciplinaria, pero al no considerar la hiperlaxitud como un problema, podría sospecharse de problemas neurológicos, falta de estimulación, "pereza" e incluso llegar a estigmatizaciones.

Una vez detectado el problema de hiperlaxitud, es necesario coordinar una adecuada estimulación psicomotora dirigida e interdisciplinaria, considerando el fortalecimiento muscular a la par de la protección articular necesaria para no generar dislocaciones o luxaciones. La meta de la intervención sería, por lo tanto, pesquisar la condición para poder promover de forma interdisciplinaria el DSM del niño afectado ${ }^{(7)}$.

En la etapa escolar, la condición HSD/hEDS no reconocida puede ocasionar estigmatizaciones en cuanto pueden ser considerados como niños "cansados, perezosos, desatentos e inactivos", etiqueta que puede ir integrándose a la autopercepción del afectado y comprometer su autoestima ${ }^{(8)}$.

Reconocer durante la atención infantil síntomas como el dolor de rodillas o de crecimiento, pasividad por cansancio, dislocaciones recurrentes, dolor de espalda, cefaleas, fatiga o dolor crónico, dificultad para escribir, bajo rendimiento escolar, torpeza motora, dificultad en la propiocepción, disautonomía progresiva, etc. permite pesquisar los HSD/hEDS y generar guías de cuidados específicos que promueven el aprendizaje, la seguridad, prevención postural, el manejo del dolor, satisfacción de necesidades como la alimentación, actividad física $y$ afectividad, tanto en el ambiente escolar como en el hogar, en colaboración conjunta con los padres, la familia y los profesores ${ }^{(9,10)}$.

Los adolescentes, por su parte, también pueden presentar disautonomía, siendo afectados por la fatiga, el cansancio, mareos, síncopes, entre otros. Sin embargo, estos síntomas en muchas ocasiones son confundidos, o más bien, relacionados como propios de la etapa y así son estigmatizados como "holgazanes", torpes o depresivos, sin haberse dado la pesquisa necesaria ${ }^{(7)}$.

En consideración de lo anterior, se debe tomar en cuenta durante los controles de salud infantil, escolar y adolescente lo siguiente: alteraciones del DSM, antecedentes de luxaciones, subluxaciones, fracturas, síntomas de disautonomía, dolor agudo o crónico y 
alteraciones psicoemocionales asociadas. Además, alteraciones ortopédicas como displasia luxante de caderas, genu varo, genu valgo, pie plano, desviaciones de la columna tales como cifosis, escoliosis o lordosis. Ante la sospecha de estar frente a un niño hiperlaxo, es conveniente realizar anamnesis y examen físico a todo el grupo familiar para fundamentar la sospecha diagnóstica y canalizar los cuidados respectivos, necesarios para los demás integrantes afectados. Lo anterior implica la coordinación con el equipo de salud para la atención y el seguimiento correspondiente. La eđucación a la familia debe contemplar la prevención de daño articular, promoción de la actividad física protectora del aparato músculo-esquelético; además, primeros auxilios en caso de luxación, subluxación o fractura u otras complicaciones más graves como síncopes, anafilaxias, entre otros. Es esencial el acompañamiento a través de las fases de adaptación a la enfermedad de larga duración, poco conocidas y hereditarias, lo que incluye la coordinación con establecimientos educacionales para la entrega de recomendaciones y, eventualmente, la educación a la comunidad: escuelas, guarderías, instituciones deportivas, etc.

Durante la segunda fase de la enfermedad ocurren cambios y se agudiza la sintomatología, estableciéndose el síndrome de dolor músculo-esquelético y visceral con signos y síntomas más notorios ${ }^{(6)}$. No obstante, muchas manifestaciones aún podrían pasar inadvertidas, por un lado a causa de una actitud escasamente preventiva que presenta en general el adulto (11), asociada a la tendencia de consultar solo cuando existe una enfermedad, $y$ también a la falta de estrategias que acerquen a este grupo etario a controles seriados de salud preventivos centrados solo en ciertas patologías, al menos en el Sistema de Salud Chileno ${ }^{(12)}$.

En esta fase, el afectado por HSD/hEDS podría padecer de sintomatología y complicaciones de diversos grados, pero a la vez debe ser altamente funcional para un modelo económico-social que implica actividades laborales o de estudios exigentes. Por tanto, el cuidado de enfermería debería incluir la realización de un examen de salud preventiva con una valoración integral de la persona, considerando no solo las enfermedades implícitas en los programas de salud, sino también la satisfacción de las necesidades para una persona de 20 años o más, como lo son el descanso y sueño, la movilidad, el dolor, el rendimiento académico o laboral y la salud sexual, entre otros. Todas las anteriores son necesidades parciales o altamente insatisfechas en el caso de los HSD/hEDS.

Se hace necesaria la escucha activa al relato que establece la persona respecto a su sintomatología y no solo la atención a los siognos y síntomas aislados, profundizando en una anamnesis próxima y remota, considerando los antecedentes familiares y la historia clínica individual, para así elaborar un plan de cuidados personalizado que determine una alimentación adecuada, observación del ciclo menstrual, salud laboral, condiciones posturales, manejo del dolor, ejercicios y fortalecimiento de la autopercepción, entre otras ${ }^{(13)}$. Asimismo, colaborar en la derivación oportuna para el diagnóstico y la evaluación interdisciplinaria con profesionales competentes en la temática: terapeuta ocupacional, kinesiólogo, fonoaudiólogo, matrona, médico, terapeutas complementarios, entre otros, y mantener el seguimiento.

En la tercera fase, uno de los síntomas más notorios y que determina de forma importante la discapacidad, junto a la fatiga, es el dolor crónico, que se manifiesta en forma de artralgias, dolor de espalda, mialogias y fibromialgias ${ }^{(6)}$.

En general todos estos dolores se presentan simultáneamente y en muchas ocasiones son mal diagnosticados como fibromialgia, fatiga crónica, depresión o hipocondría, entre otros ${ }^{(2)}$. Lo que produce un retraso en el manejo integrado de los afectados, aportando al agravamiento de las complicaciones $y$ de la discapacidad progresiva. Debido al sub-diagnóstico, los expertos recomiendan que frente a cada paciente que presenta un dolor crónico debiera pensarse en la posibilidad de HSD/hEDS y examinarle para hiperlaxitud articular ${ }^{(2)}$.

Desde la presente reflexión, el dolor como síntoma, es decir, como percepción subjetiva no medible, es la manifestación que produce mayor incomprensión de parte del medio que rodea a los afectados, sean los médicos tratantes, familiares, colegas de trabajo, amigos, etc.

Es así que a los no afectados, muchas veces se les hace difícil comprender lo que sionifica el dolor para quien lo padece. Esta incomprensión puede intensificarse además, porque los afectados suelen ser aún adultos 
jóvenes, en plena etapa de vida laboral y familiar, tendiéndose a relacionar la experiencia del dolor y la fatiga a situaciones de estrés, de incapacidad de manejo de situaciones exigentes o a la falta de capacidad del rendimiento.

Lo anterior se complejiza cuando el diagnóstico todavía no está hecho y la persona afectada no entiende lo que le está pasando. Aunque también ocurre cuando ya lo conoce, porque debe entrar en una constante explicación de su condición a terceros, ya que en la perspectiva de estos últimos pudiese parecer invisible, porque podría no requerir de elementos identificatorios como por ejemplo, una silla de ruedas $u$ otras ayudas técnicas ${ }^{(1)}$.

La incomprensión por la discapacidad causada por el dolor, la fatiga y la disautonomía, podría constituirse como uno de los factores emocionales más estresantes para los afectados, la cual se suma al estrés que, de por sí, producen los propios síntomas y el esfuerzo que se hace para "funcionar» lo mejor posible en la vida diaria. Es de esperar que como consecuencia del dolor crónico y las demás complicaciones sistémicas y osteoarticulares, se produce una importante disminución en la calidad de vida ${ }^{(1)}$, ya que la discapacidad se presenta no solo en el plano laboral, sino en las actividades simples de la vida diaria como cocinar, asear la casa, vestirse, ducharse, usar un computador y también recrearse. La dimensión social podría verse afectada por no poder asistir a convivencias, eventos culturales, fiestas, etc.; la calidad de vida afectiva depende en gran medida de la comprensión que generan personas cercanas al afectado.

Entre los cuidados de enfermería necesarios en esta etapa, podemos mencionar escuchar y dar crédito a la narrativa personal, colaborar en la búsqueda de diagnóstico, derivación y seguimiento, elaborar conjuntamente estrategias de autocuidado diario como saber pedir ayuda, adaptación de rutinas, alimentación, reacomodación del proyecto de vida, ejercicios, asistencia a los grupos de autoayuda de afectados, prevención de automedicación, acompañamiento en el luto por pérdidas percibidas, educación sobre la enfermedad y farmacología y derivación dentro del equipo de salud $y$, potencialmente, asesoría legal y económica. En caso de hospitalización, deberán brindarse los respectivos cuidados según especialidad.

\section{CONSIDERACIONES FINALES}

Para concluir, se enfatiza en la importancia del rol del profesional enfermero en la gestión del cuidado de los niños y adultos que viven con este serio problema de salud que constituyen los HSD/hEDS, los cuales, probablemente, afectan a muchas más personas de lo que se cree en la actualidad. Es primordial tomar nota de su existencia y revisar cómo cada disciplina de la salud, en particular y en conjunto, pueden aportar a la pesquisa, al manejo y cuidado de las personas afectadas. En este sentido, para la enfermería, se proponen actividades de promoción de la salud de acuerdo con las manifestaciones particulares de cada afectado, coordinar el manejo interdisciplinario, integrar los conocimientos teóricos y prácticos sobre los trastornos del tejido conectivo en la formación de pregrado y posgrado de enfermería y de investigación disciplinar e interdisciplinar.

Como desafíos, quedan evitar la parcelación de la atención de personas con HSD/hEDS, fomentando la integración de las manifestaciones para ir comprendiendo las características del espectro y síndrome, definir estrategias interdisciplinarias de atención $\varnothing$ flujogramas, tanto a nivel público como privado, además de hacer visible y cuantificable la condición mediante la investigación para avanzar en su pesquisa oportuna.

\section{FINANCIAMIENTO}

Sin financiamiento. Sin embargo autor Camilo Guerrero-Nancuante cursa estudios de postorado financiado por CONICYT, Programa de formación de capital humano avanzado/Magíster Nacional/2018-22181048.

\section{CONFLICTOS DE INTERESES}

Ninguno por declarar.

\section{REFERENCIAS BIBLIOGRÁFICAS}

1. Berglund $B$, Pettersson $C$, Pigg $M$, Kristiansson P. Self-reported quality of life, anxiety and depression in individuals with Ehlers-Danlos syndrome (EDS): a questionnaire study. BMC Musculoskeletal 
Disorders [Internet]. 2015,16:89. [citado 23 de agosto de 2018];16(1):89. Disponible en: https://doi. org/10.1186/s12891-015-0549-7

2. Gazit Y, Jacob G, Grahame R. Ehlers-Danlos Syndrome-Hypermobility Type: A Much Neglected Multisystemic Disorder. Rambam Maimonides Med J. 2016,7(4). DOI 10.5041/RMMJ.10261.

3. Castori M, Tinkle B, Levy H, Grahame R, Malfait F, Hakim A. A framework for the classification of joint hypermobility and related conditions. AJMG. 2017,175(1):148-57. DOI 10.1002/ajmg.c.31539.

4. Juul-Kristensen B, Schmedling K, Rombaut L, Lund H, Engelbert RHH. Measurement properties of clinical assessment methods for classifying generalized joint hypermobility-A systematic review. Am J Med Genet C Semin Med Genet. 2017,175(1):116-47. DOI 10.1002/ ajmg.c.31540.

5. Malfait F, Francomano C, Byers P, Belmont J, Berglund B, Black J, et al. The 2017 international classification of the Ehlers-Danlos syndromes. AJMG. 2017,175(1):826. DOI 10.1002/ajmg.c.31552.

6. Castori M, Morlino S, Celletti C, Ghibellini G, Bruschini M, Grammatico P, et al. Re-writing the natural history of pain and related symptoms in the joint hypermobility syndrome/Ehlers-Danlos syndrome, hypermobility type. AJMG. 2013,161A(12):2989-3004. DOI 10.1002/ajmg.a.36315.
7. Skills for action [internet]. Cape Town: Skills for action; 2015 [Acceso 23 de Agosto de 2018]. Disponible en: https://skillsforaction.com/infant-joint-hypermobility

8. Murray K. Hypermobility disorders in children and adolescents. Best Pract Res Clin Rheumatol. 2006;20(2):329-51. DOI 10.1016/j.berh.2005.12.003.

9. The Ehlers-Danlos Society. An educator's guide Meeting the Needs of the Ehlers-Danlos Child: a parent's guide Helping Your Child Succeed at School [Internet]. [Consultado 23 de Ago. de 2018]. Disponible en: http://cort.as/-LQuU

10. Cattalini M, Khubchandani R, Cimaz R. When flexibility is not necessarily a virtue: a review of hypermobility syndromes and chronic or recurrent musculoskeletal pain in children. Pediatr Rheumatol Online J. 2015,13(40). DOI 10.1186/s12969-015-0039-3.

11. Hernán M, Fernández A, Ramos M. La salud de los jóvenes. Gac Sanit. 2004, 18(4):47-55.

12. Miranda Hiriart G, Saffie Gatica X. Pacientes policonsultantes: ¿un síntoma del sistema de salud en Chile?. Acta Bioeth. 2014;20(2):215-23. DOI 10.4067/S1726569X2014000200009.

13. Larrate JM, Martin RS, Alejo FM. El síndrome de hiperlaxitud articular en la práctica clínica diaria. Rev Cuba Reumatol. 2013;15(1):36-41. 